\title{
Emergent Magnetic Phases in Pressure-Tuned van der Waals Antiferromagnet $\mathrm{FePS}_{3}$
}

\author{
Matthew J. Coak $\odot,{ }^{1,2, *}$ David M. Jarvis $\odot,{ }^{1}$ Hayrullo Hamidov $\odot,{ }^{1,3,4}$ Andrew R. Wildes $\odot,{ }^{5}$ Joseph A. M. Paddison $\odot,{ }^{6,1}$ \\ Cheng Liu $\odot,{ }^{1}$ Charles R. S. Haines $\odot,{ }^{7,1}$ Ngoc T. Dang $\odot,{ }^{8,9}$ Sergey E. Kichanov $\odot,{ }^{10}$ Boris N. Savenko, ${ }^{10}$ \\ Sungmin Lee, ${ }^{11,12}$ Marie Kratochvílová $\odot,{ }^{11-13}$ Stefan Klotz $\odot,{ }^{14}$ Thomas C. Hansen $\odot,{ }^{5}$ \\ Denis P. Kozlenko® ${ }^{10}$ Je-Geun Park $\oplus^{11,12,15}$ and Siddharth S. Saxena $\oplus^{1,4, \uparrow}$ \\ ${ }^{1}$ Cavendish Laboratory, University of Cambridge, \\ J. J. Thomson Avenue, Cambridge CB3 OHE, United Kingdom \\ ${ }^{2}$ Department of Physics, University of Warwick, Gibbet Hill Road, Coventry CV4 7AL, United Kingdom \\ ${ }^{3}$ Navoi State Mining Institute, 27 Galaba Avenue, Navoi, Uzbekistan \\ ${ }^{4}$ National University of Science and Technology "MISiS," Leninsky Prospekt 4, Moscow 119049, Russia \\ ${ }^{5}$ Institut Laue-Langevin, 71 Avenue des Martyrs, 38000 Grenoble, France \\ ${ }^{6}$ Materials Science and Technology Division, Oak Ridge National Laboratory, \\ Oak Ridge, Tennessee 37831, USA \\ ${ }^{7}$ Department of Earth Sciences, University of Cambridge, \\ Downing Street, Cambridge CB2 3EQ, United Kingdom \\ ${ }^{8}$ Institute of Research and Development, Duy Tan University, 550000 Da Nang, Vietnam \\ ${ }^{9}$ Faculty of Natural Sciences, Duy Tan University, 550000 Da Nang, Vietnam \\ ${ }^{10}$ Frank Laboratory of Neutron Physics, Joint Institute for Nuclear Research, 141980 Dubna, Russia \\ ${ }^{11}$ Center for Correlated Electron Systems, Institute for Basic Science, Seoul 08826, Republic of Korea \\ ${ }^{12}$ Department of Physics and Astronomy, Seoul National University, Seoul 08826, Republic of Korea \\ ${ }^{13}$ Faculty of Mathematics and Physics, Department of Condensed Matter Physics, Charles University, \\ Prague, Czech Republic \\ ${ }^{14}$ Sorbonne Université, IMPMC, CNRS, UMR 7590, 4 Place Jussieu, 75252 Paris, France \\ ${ }^{15}$ Center for Quantum Materials, Seoul National University, Seoul 08826, Republic of Korea
}

(Received 9 April 2020; revised 15 September 2020; accepted 28 October 2020; published 5 February 2021)

Layered van der Waals 2D magnetic materials are of great interest in fundamental condensed-matter physics research, as well as for potential applications in spintronics and device physics. We present neutron powder diffraction data using new ultrahigh-pressure techniques to measure the magnetic structure of Mottinsulating 2D honeycomb antiferromagnet $\mathrm{FePS}_{3}$ at pressures up to $183 \mathrm{kbar}$ and temperatures down to $80 \mathrm{~K}$. These data are complemented by high-pressure magnetometry and reverse Monte Carlo modeling of the spin configurations. As pressure is applied, the previously measured ambient-pressure magnetic order switches from an antiferromagnetic to a ferromagnetic interplanar interaction and from 2D-like to 3D-like character. The overall antiferromagnetic structure within the $a b$ planes, ferromagnetic chains antiferromagnetically coupled, is preserved, but the magnetic propagation vector is altered from $\mathbf{k}=\left(0,1, \frac{1}{2}\right)$ to $\mathbf{k}=(0,1,0)$, a halving of the magnetic unit cell size. At higher pressures, coincident with the second structural transition and the insulator-metal transition in this compound, we observe a suppression of this long-range order and emergence of a form of magnetic short-range order which survives above room temperature. Reverse Monte Carlo fitting suggests this phase to be a short-ranged version of the original ambient-pressure structure - with the Fe moment size remaining of similar magnitude and with a return to antiferromagnetic interplanar correlations. The persistence of magnetism well into the HP-II metallic state is an observation in contradiction with previous $\mathrm{x}$-ray spectroscopy results which suggest a spin-crossover transition.

DOI: 10.1103/PhysRevX.11.011024

Subject Areas: Condensed Matter Physics, Magnetism, Strongly Correlated Materials

\footnotetext{
*Corresponding author. matthew.coak@warwick.ac.uk

Corresponding author. sss21@cam.ac.uk
}

Published by the American Physical Society under the terms of the Creative Commons Attribution 4.0 International license. Further distribution of this work must maintain attribution to the author(s) and the published article's title, journal citation, and DOI. 


\section{INTRODUCTION}

The study of low-dimensional magnetism has long been of great interest in fundamental condensed-matter physics and has led to numerous applications. Layered magnetic van der Waals materials-compounds with planes of magnetic ions separated by wide van der Waals bonded gaps-provide a wide assortment of ideal systems for investigating 2D magnetism. A particularly powerful approach to investigating the physics in low-dimensional magnetic systems is to tune the interactions and dimensionality of the lattice and to explore how the magnetic and electronic behaviors of the material evolve. A large volume of recent work focuses on thickness control, to tune toward the "true 2D" limit of the atomic monolayer [1-5], with many interesting results. A complementary, and critical, approach is to tune the magnetic interactions from 2D to 3D by applying hydrostatic pressure - in a van der Waals material, applying pressure predominantly compresses the weakly bonded interplanar separation and pushes the lattice toward a 3D character. This approach can be viewed as moving from the "quasi-2D" starting point in the opposite direction to a thickness dependence study and addresses the same fundamental physics questions. Both approaches are needed for a full understanding of dimensionality in these fascinating systems to be accomplished. The use of pressure as a tuning parameter unlocks a cleaner and more controllable approach to map complete phase diagrams than methods such as chemical substitution or uniaxial strains.

The group of van der Waals (vdW) antiferromagnets $T M \mathrm{PS}_{3}$ [6-10], with $T M$ a first-row transition metal, have been studied for some time as near-ideal 2D magnetic systems [11-26]. These compounds were first discovered in the 1890s and rigorously reported on in the 1980s [27-34]. They all share the same basic crystal structure, shown in Fig. $1-$ a $C 2 / m$ monoclinic cell with a honeycomb arrangement of metal ions. These magnetic sites are arranged in slightly distorted hexagons in the $a b$ plane, separated by wide vdW gaps along the $c$ axis. Intersite exchange both within the crystal planes and between layers is mediated through $\mathrm{P}_{2} \mathrm{~S}_{6}$ clusters surrounding the metal honeycombs, and there is significant competition between several exchange interactions of comparable strengths. Substituting the $T M^{2+}$ ion across the family leads to a wide variety of different magnetic order types and anisotropies while keeping the same crystal structure, allowing clear and clean comparisons of, for example, Heisenberg versus Ising magnetic Hamiltonians in the 2D limit. (a)

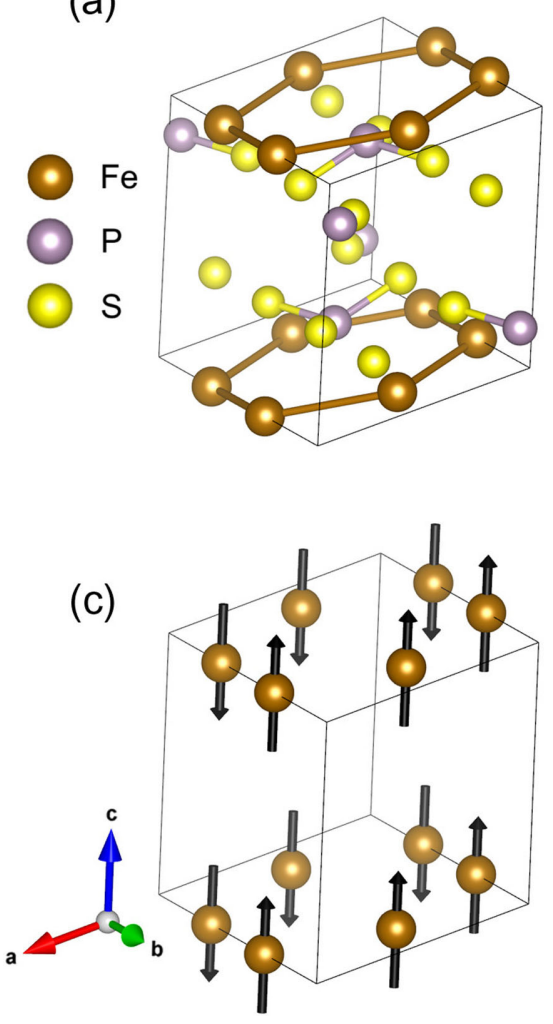

(b)

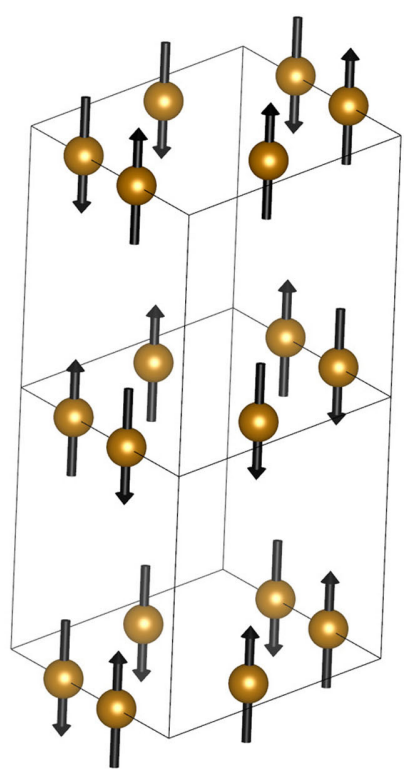

FIG. 1. Crystal and magnetic structures of ambient-pressure FePS 3 . (a) The monoclinic $C 2 / m$ crystal structure [27]. (b) The magnetic cell of $\mathrm{FePS}_{3}$ with the ambient-pressure $\mathbf{k}=\left(0,1, \frac{1}{2}\right)$ propagation vector-overall antiferromagnetic order comprised of zigzag chains along $a$, antiferromagnetically coupled to their neighbors and between the crystal planes [11]. (c) The magnetic unit cell with $\mathbf{k}=(0,1,0)$ propagation vector, where planes are instead ferromagnetically aligned. 
In recent years, we have conducted a range of studies into the structural and transport properties of $\mathrm{FePS}_{3}, \mathrm{VPS}_{3}$, $\mathrm{NiPS}_{3}$, and $\mathrm{MnPS}_{3}$ under pressure [35-39]. This work reveals two structural transitions with increasing pressure common to all these materials, a shear motion of the $a b$ planes along the $a$ axis, which brings the $\beta$ unit cell angle to close to $90^{\circ}$ while remaining a monoclinic phase (structure HP-I), and then at higher pressures a collapse of the interplanar separation and a transition to a trigonal symmetry group (HP-II) [35]. In $\mathrm{FePS}_{3}$, the HP-I to HP-II structural transition is accompanied by an insulator-metal transition, as electron overlap and hopping are greatly enhanced. This transition is a clear move from $2 \mathrm{D}$ toward a $3 \mathrm{D}$ character, as well as a change in the symmetry of the lattice, and can reasonably be expected to have a significant effect on the magnetism in this material. In the sister compound $\mathrm{FePSe}_{3}$, continuing to increase pressure following the insulator-metal transition leads to the formation of a superconducting phase [40] from the Mott-insulating 2D antiferromagnetic ambient state.

$\mathrm{FePS}_{3}$ is an Ising antiferromagnet with a Néel temperature $T_{N}$ of around $118 \mathrm{~K}$ [12]. Figure 1(b) shows the established ambient-pressure magnetic structure [11,13]. Zigzag ferromagnetic chains are formed along $a$. The moments are parallel to $c^{*}$ ( $c^{*}$ is perpendicular to the crystal planes). The chains are antiferromagnetically coupled both in plane, along $b$, and between the planes, along $c$, giving the resulting magnetic propagation vector $\mathbf{k}=\left(0,1, \frac{1}{2}\right)$. However, the mere act of grinding a powder sample appears to introduce enough dislocations and stacking faults to essentially randomize longrange order along $c^{*}$, resulting in rodlike features in neutron magnetic diffraction patterns parallel to the $c^{*}$ axis and centered at the $\mathbf{k}=(0,1,0)$ propagation vector [11,19]. Single-crystal data do not display this behavior but instead sharp, long-ranged 3D Bragg peaks [11]. A propagation vector of $\mathbf{k}=(0,1,0)$ corresponds to the same in-plane magnetic structure as the $\mathbf{k}=\left(0,1, \frac{1}{2}\right)$ but with the planes ferromagnetically coupled, as shown in Fig. 1(c).

In this work, we report the first study of the magnetic structure under high pressure in the $T M \mathrm{PS}_{3}$ materials, through powder neutron diffraction. The development of double-toroidal sintered diamond anvils for the ParisEdinburgh high-pressure press allows for measurements to be carried out above $100 \mathrm{kbar}$ - we are able to observe the evolution of the magnetic structure well into the metallic HP-II phase of $\mathrm{FePS}_{3}$ up to 183 kbar.

\section{METHODS}

Powder neutron diffraction patterns are collected on beam line D20 at the Institut Laue-Langevin (ILL), France [41]. Data are collected during two separate experiments. The first uses a Paris-Edinburgh-type pressure cell press [42] with cubic boron nitride (cBN) anvils [43]. The second employs double-toroidal sintered diamond anvils in the same press [44], to achieve significantly higher pressure values [45]. A single-crystal sample is ground into a fine powder in liquid nitrogen and in an argon glovebox to mitigate preferred orientation and water uptake. This powder is packed into the two halves of a $\mathrm{Ti} / \mathrm{Zr}$ null scattering spherical gasket [46] and this placed between the anvils. The powder is wetted with $4: 1$ deuterated methanol-ethanol mixture to serve as a hydrostatic pressure medium. For the first experiment, a piece of lead is placed in the center of the sample volume to act as a pressure gauge via its equation of state [47]. In the later experiment using sintered diamond anvils, no pressure gauge is used, as the pressure dependence of the lattice parameters of the sample are known (at room temperature) [35], and so the pressure can be determined from the sample peak positions. The position of the 001 peak is used for pressures up to $103 \mathrm{kbar}$, as this position is highly sensitive to pressure, and then the 131 at pressures beyond this value, as this position then becomes more sensitive in the high-pressure structure. The pressures measured in the first experiment are 0,20,49,72, and $80 \mathrm{kbar}$ and in the second 33, 78, 103, 157, and 183 kbar. Typical uncertainty on pressure values is a few kilobars. All pressure changes are made above $300 \mathrm{~K}$, and then the cell is cooled to $80 \mathrm{~K}$ and data are taken at this base temperature and during warming, under constant load.

The wavelength of neutrons used is $2.42 \AA$, via a graphite monochromator. Peaks in the neutron diffraction patterns originating from the anvils used, and the $\mathrm{Pb}$ manometer when present, are seen in addition to the sample signals. The diamond peaks are easily identified, as they do not noticeably shift with pressure. With the diamond anvil measurements, a prominent peak at around $72^{\circ}$ of $2 \theta$ is observed (removed from the presented datasets) but also a series of peaks at smaller $Q$. These do not move and, hence, must be attributed to the diamonds or other external cell environment and could, in fact, be indexed as originating from diamond but with a wavelength of $1 / 3$ that used in the measurement. The peaks are extremely weak compared to the principal diamond peak, so we conclude that a small weight of $\lambda / 3$ neutrons are able to pass the graphite filter designed to suppress $\lambda / n$ wavelengths and lead to a measurable diamond diffraction pattern. These peaks are all marked as "diamond" in the presented data and can, of course, be ignored.

Additional neutron powder diffraction measurements at pressures up to $49 \mathrm{kbar}$ are performed from 15 to $290 \mathrm{~K}$ on the DN-12 diffractometer, IBR-2 pulsed reactor, Joint Institute for Nuclear Research (JINR), Russia [48]. A powder sample of volume approximately $2 \mathrm{~mm}^{3}$ is loaded into a sapphire anvil high-pressure cell with culets of $4 \mathrm{~mm}$ diameter [49]. Spherical cavities with a diameter of $2 \mathrm{~mm}$ are drilled at the culet centers to encourage quasihydrostatic pressure distribution at the sample surface. No pressure-transmitting medium is used. The diffraction 
patterns are collected at scattering angles of $90^{\circ}$ and $45^{\circ}$ with resolution $\Delta d / d=0.015$ and 0.022 , respectively. The pressure is measured using the ruby fluorescence technique [50]. Pressure gradients are less than $10 \%$ with respect to average pressure values. The diffraction patterns are analyzed by the Rietveld method using the Fullprof software suite [51].

The dc magnetization data are measured in an Magnetic Properties Measurement System superconducting quantum interference device magnetometer, Quantum Design, in an applied field of $0.1 \mathrm{~T}$. The pressure cell used is a pistoncylinder clamp cell from Camcool Research Limited, with a Daphne oil pressure medium and a lead manometer. Measurements are carried out to the maximum achievable pressure of cells of this type-around $10 \mathrm{kbar}$. Higher pressures require a diamond anvil cell, where the tiny sample volume precludes resolving the small signal of an antiferromagnet like $\mathrm{FePS}_{3}$.

Reverse Monte Carlo fits are conducted in the Spinvert program $[52,53]$ to model short-range order in the highestpressure data. A supercell of $10 \times 10 \times 10$ unit cells is used, and the previously solved HP-II crystal structure, with lattice parameters refined from the neutron data collected at this pressure.

\section{RESULTS}

The initial effect of pressure on the antiferromagnetic order in $\mathrm{FePS}_{3}$ is to raise the value of $T_{N}$, as shown in
Fig. 2. The inset shows magnetization curves measured under pressure around the antiferromagnetic transitionthese are clearly and continuously shifted upward in temperature as pressure is increased. $T_{N}$ is extracted from differentiating these curves and, additionally, from integrated magnetic peak intensities of the neutron diffraction patterns - these values are used to build the temperature-pressure phase diagram shown in the main figure. The value of $T_{N}$ increases linearly with pressure, initially at a rate of $0.77 \mathrm{~K} \cdot \mathrm{kbar}^{-1}$. Pressure pushes the crystal planes together and, hence, allows larger interlayer exchange, so it is consistent to see a strengthening of the magnetic order and it stabilizing at higher temperatures. At approximately $20 \mathrm{kbar}$, there appears to be a change of slope, and thereafter the rate is reduced to $0.32 \mathrm{~K} \cdot \mathrm{kbar}^{-1}$. This pressure corresponds to the value at which (at room temperature) the ambient-pressure structure begins to (gradually) cross over to the HP-I structure [35]. A stiffening of the rate of change as the crystal enters the HP-I phase, which has stronger interplanar coordination, is again consistent and is reminiscent of the stiffening of vibrational modes seen in $\mathrm{V}_{0.9} \mathrm{PS}_{3}$ [37]. At higher pressures above $100 \mathrm{kbar}$, as discussed in the later sections, the magnetic order takes on completely different forms, and the linear trend is no longer a valid description.

The ambient-pressure neutron powder diffraction patterns, obtained within the cBN single toroidal cell, are shown in Fig. 3 for temperatures of 300 and $80 \mathrm{~K}$ (the

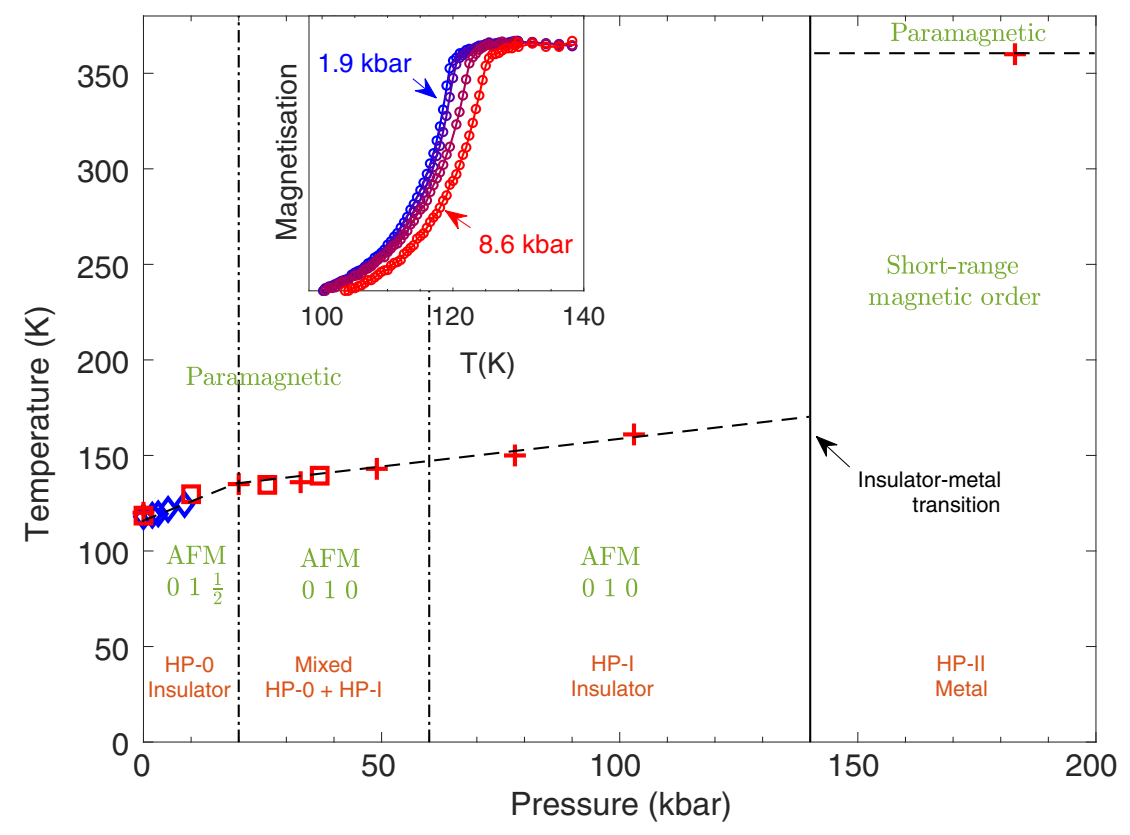

FIG. 2. Pressure-temperature phase diagram for $\mathrm{FePS}_{3}$ constructed from data found in this work and the structural results from Ref. [35]. Data points display extracted temperatures of magnetic transitions plotted against pressure. Blue circles show values extracted from magnetization measurements, and red crosses and squares from integrating the magnetic peaks and features in the neutron powder patterns from the ILL and JINR data, respectively. Dashed lines delineating the AFM state are fits to the data points; gradients are stated in the text. Vertical lines on the phase diagram designate the pressures at which structural transitions occur. The inset shows normalized magnetization data plotted against temperature for increasing pressures. 


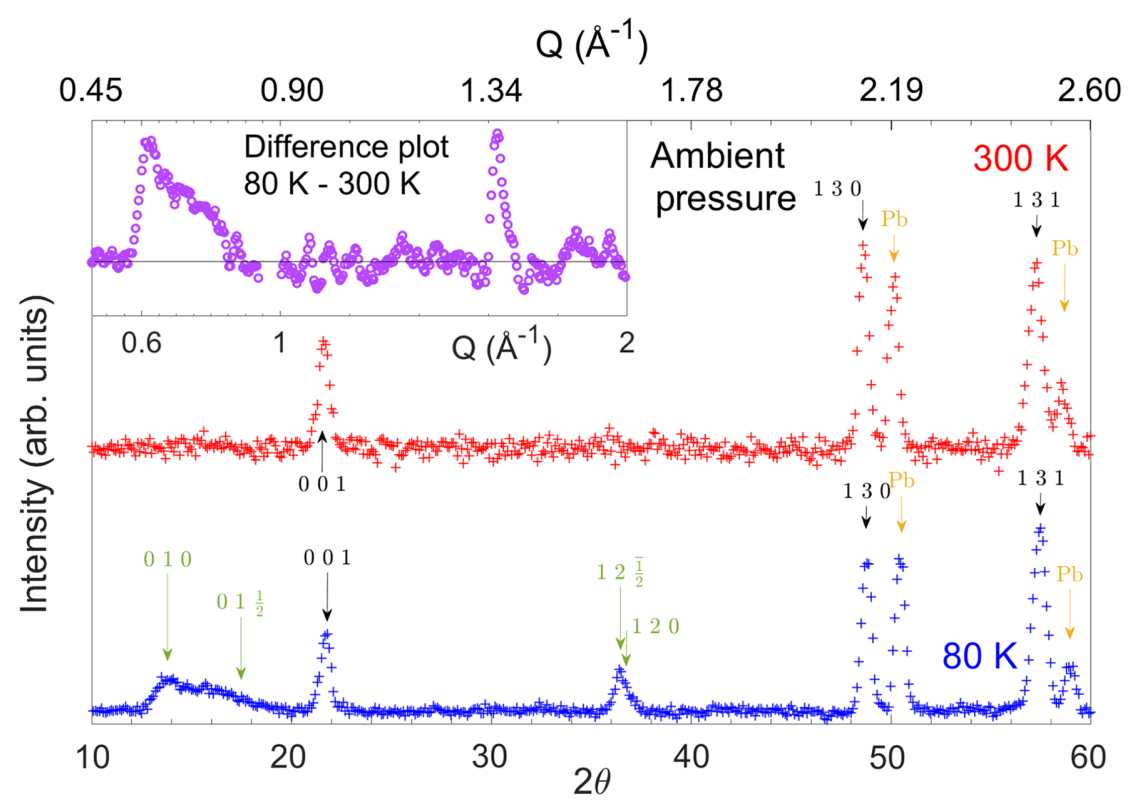

FIG. 3. Powder neutron diffraction patterns of $\mathrm{FePS}_{3}$ at ambient pressure at 80 and $300 \mathrm{~K}$ taken on D20, ILL. Nuclear and magnetic Bragg peaks are marked (magnetic in green) as indexed in the monoclinic low-pressure phase. The magnetic propagation vector at ambient pressure is $\mathbf{k}=\left(0,1, \frac{1}{2}\right)$, but strain and stacking faults in the crystal due to grinding lead to $2 \mathrm{D}$ rodlike ordering with vector $\mathbf{k}=(0,1,0)$, as described in Refs. $[11,13,19]$. This ordering causes the broad and asymmetric peak visible at small $Q$ in the $80 \mathrm{~K}$ data. An additional magnetic feature at higher $Q$ can be indexed as the 120 and/or the $12 \frac{1}{2}$. The broad diffuse background of the methanolethanol pressure medium is subtracted from all plots shown. The inset shows the result of subtracting the $300 \mathrm{~K}$ data from the $80 \mathrm{~K}$ plotted against $Q$ to reveal the solely magnetic signal.

lowest temperature measured). The patterns are normalized, and a diffuse background due predominantly to the methanol-ethanol pressure medium is subtracted from each dataset by fitting a cubic spline function to the data between peaks and features. Below $T_{N}$, the magnetic peaks and features indexed and labeled in green in the figure are seen to appear. These match the form reported in previous work $[11,19]$. A broad asymmetric feature, indicative of order of a 2D nature, spanning 010 to $01 \frac{1}{2}$ is the principal magnetic feature. An additional peak at higher $Q$ can be indexed either as the 120 , corresponding to a combination of the magnetic 010 Bragg peak with the nuclear 110 Bragg peak, or as the $12 \frac{1}{2}$, a combination of the magnetic 0 $1 \frac{1}{2}$ and nuclear $11 \overline{1}$ peaks — these have extremely similar $d$ spacings.

When pressure is applied, the magnetic structure shows a clear change, as illustrated in the 78 kbar example shown in Fig. 4. At this pressure, $\mathrm{FePS}_{3}$ is in its HP-I structural phase, where the crystal planes are sheared along the $a$ axis and $\beta$ is close to $90^{\circ}$. A single sharp magnetic Bragg peak is observed at 010 , replacing the rodlike scattering observed at ambient pressure. A move to a clear long-range magnetic order is consistent with the expected driving toward a 3D nature of the crystal. A $\mathbf{k}=(0,1,0)$ magnetic propagation vector means that, as shown in Fig. 1, the magnetic unit cell is now halved in volume, and the crystal planes are coupled ferromagnetically rather than antiferromagnetically. With the shear motion in the ambient-HP-I structural transition, it is reasonable to expect changes to the interlayer exchange pathways, consistent with this result. Only a minor change in exchange pathways is required to make this change, as the interlayer exchange under ambient pressure is very small (and negative) [11] so could easily be driven to be small and positive. This result is additionally consistent with the appearance of the rodlike features centered at 010 in the ambient-pressure patterns upon grinding-if the interlayer exchange is sensitive to shear along the $a$ axis and can easily be flipped in sign, grinding the sample drives it to adopt a $\mathbf{k}=(0,1,0)$ propagation vector. Previous studies at ambient pressure $[11,19]$ suggest that grinding the sample introduces disorder between the planes that affect the long-ranged order along $c^{*}$, making the magnetism more two-dimensional. Our results show that the pressure or strain effects of the grinding may also change the sign of the interplanar magnetic correlations for parts of the sample. The interplay of strain, structure, and magnetic order shown here is potentially of great significance in the rapidly growing field of heterostructure and device physics employing these materials, where strains are either an inescapable issue or a powerful engineering opportunity.

Data taken in sapphire anvil cells at JINR, Russia, show more detail of the described low-pressure evolution of the magnetic structure (Fig. 5). In these data, the ambient- 


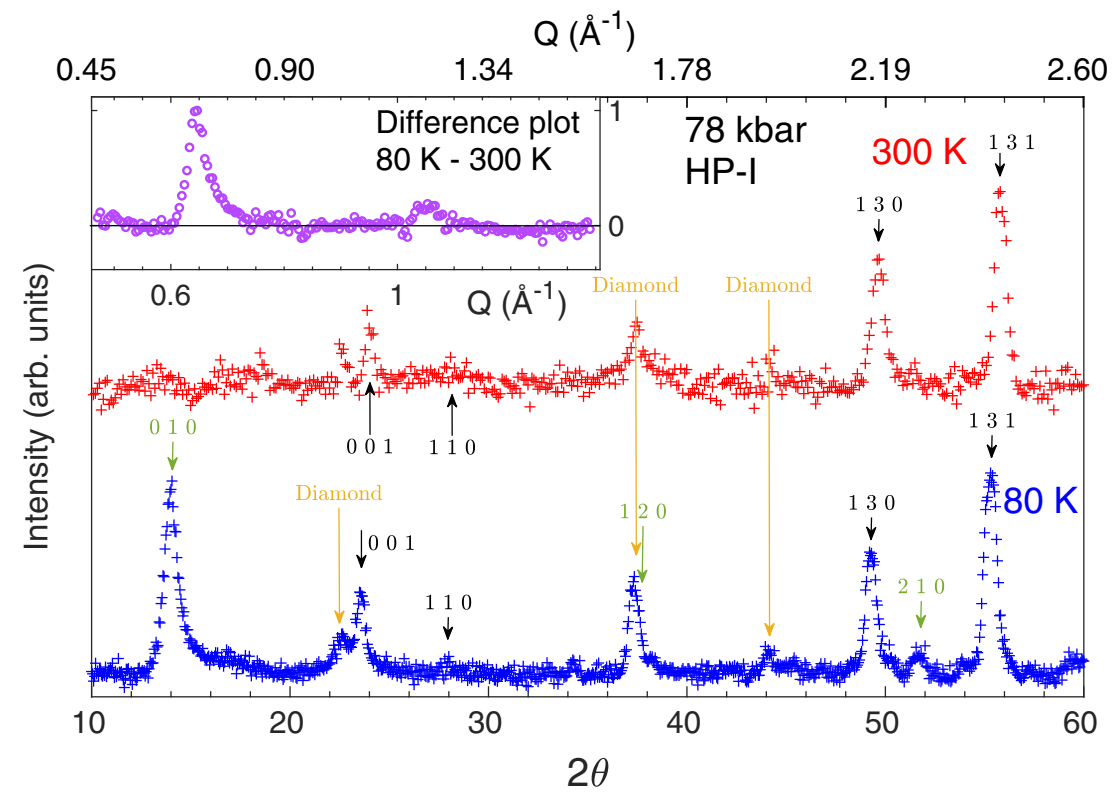

FIG. 4. Powder neutron diffraction patterns of $\mathrm{FePS}_{3}$ at $78 \mathrm{kbar}$, at 80 and $300 \mathrm{~K}$ taken on D20, ILL. Nuclear and magnetic Bragg peaks are marked as indexed in the monoclinic HP-I phase, and peaks due to the diamond anvils are marked in orange. The magnetic propagation vector is $\mathbf{k}=(0,1,0)$ and is characterized by a sharp symmetric Bragg peak. Additional magnetic peaks are seen at 120 $\left[\mathbf{k}=(0,1,0)+\left(\begin{array}{ll}1 & 1\end{array}\right)\right]$ and $210[\mathbf{k}=(0,1,0)+(200)]$ from the combination of magnetic and nuclear scattering. The inset shows the result of subtracting the $300 \mathrm{~K}$ data from the $80 \mathrm{~K}$ plotted against $Q$.

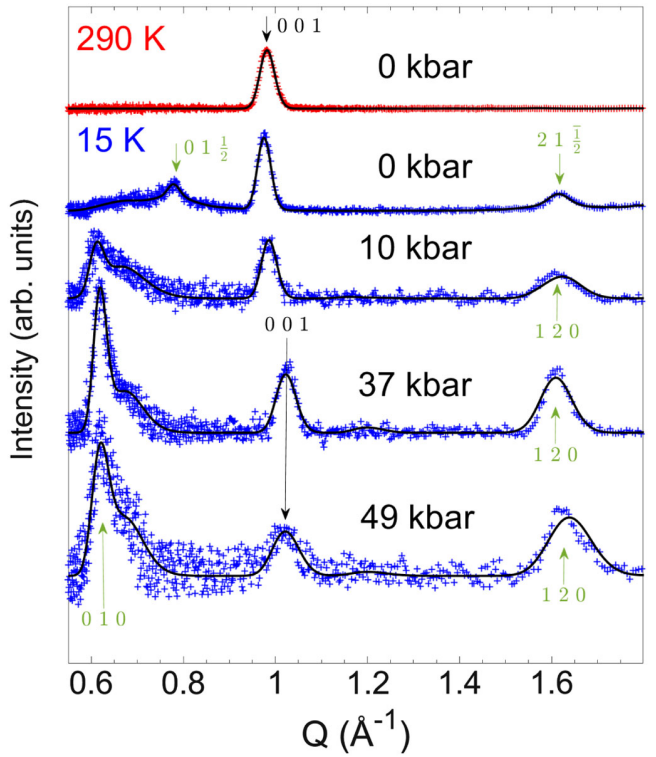

FIG. 5. Neutron diffraction patterns of $\mathrm{FePS}_{3}$, measured at selected pressures up to $49 \mathrm{kbar}$ at room temperature and $15 \mathrm{~K}$. These data are taken in sapphire anvil cells at JINR, Russia. Crosses show the experimental data and black solid lines the results of refinements of the data by the Rietveld method. In the ambient-pressure data shown at the top, the magnetic propagation vector is much more clearly $\mathbf{k}=\left(0,1, \frac{1}{2}\right)$. This result is rapidly suppressed in favor of $\mathbf{k}=(0,1,0)$, with an additional shoulder to the right of this peak suggestive of remnants of 2D-like ordering. Between 10 and $37 \mathrm{kbar}$, the 001 nuclear Bragg peak clearly shifts due to the ambient-HP-I structural transition. pressure magnetic order is more clearly characterized by the $\mathbf{k}=\left(0,1, \frac{1}{2}\right)$ propagation vector than in the data shown in Fig. 3, with an almost symmetric 3D-like Bragg peak at this position. This result can be explained by less grinding and shear strain during preparation of the sample than in the data taken at ILL. Reitveld refinements of these data yield a magnetic moment at ambient pressure of 5.40(5) $\mu_{B}$ per Fe, in line with previously published results [13]. Between the 10 and $37 \mathrm{kbar}$ datasets shown, the ambient to HP-I structural transition occurs, shifting the position of the 001 nuclear peak. Before this transition occurs, by $10 \mathrm{kbar}$, the magnetic Bragg peak at $01 \frac{1}{2}$ has been suppressed in favor of a growing peak at the 010 position, which reaches its full strength by $37 \mathrm{kbar}$. This result is consistent with the ILL data. Clearly visible in these data, however, is a shoulder or broad bump to the right of the 010 peak. We interpret this bump as an asymmetric peak shape of the 010 due to 2D-like magnetic ordering, as previously discussed in the context of the ambient-pressure data in Fig. 3. This result suggests the crystal, while gaining stronger interplanar interactions and moving toward three-dimensionality, still maintains a quasi-2D character at these pressures.

At the highest pressures, within the HP-II trigonal structural phase and following metallization, we observe another change in the magnetic ordering of $\mathrm{FePS}_{3}$. Figure 6 shows the neutron diffraction patterns at room temperature and at $80 \mathrm{~K}$ for $183 \mathrm{kbar}$, the highest pressure measured. At this point, the data show a broad hump or feature at low 


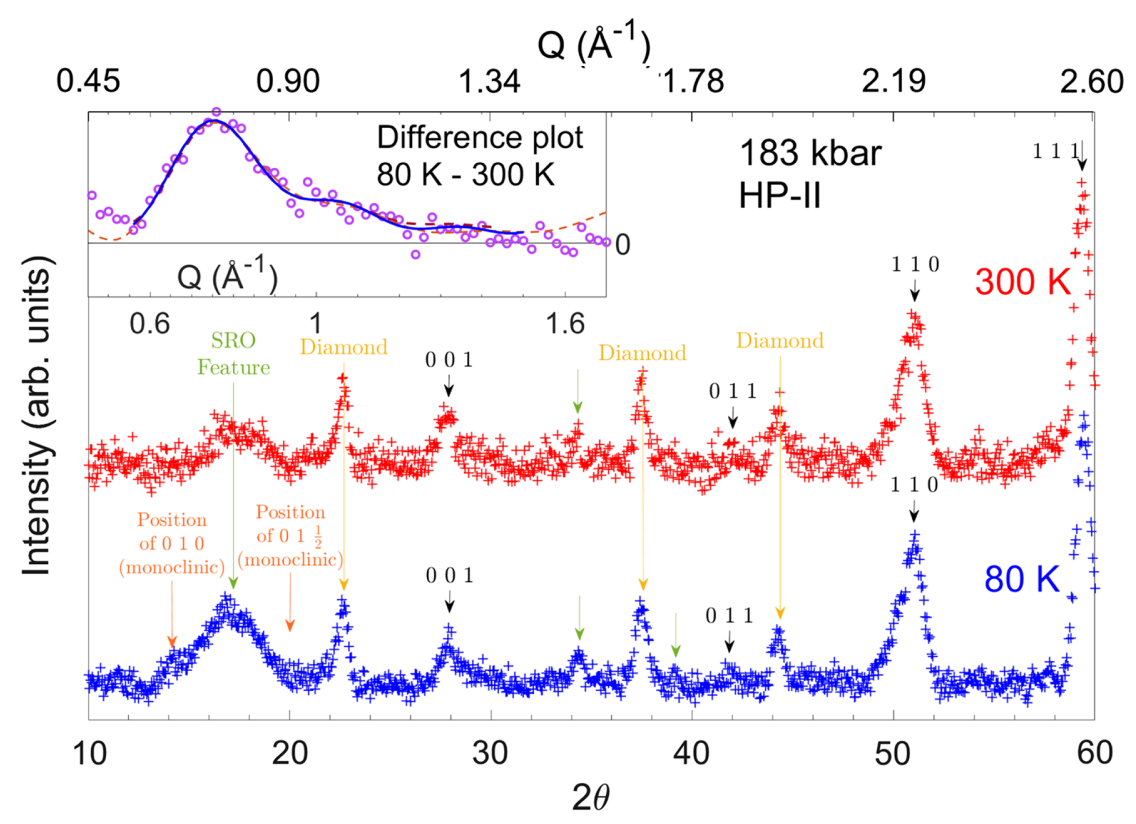

FIG. 6. Powder neutron diffraction patterns of $\mathrm{FePS}_{3}$ at $183 \mathrm{kbar}$ at 80 and $300 \mathrm{~K}$ taken on D20, ILL. Peaks originating from the sintered diamond anvils are marked in orange. Nuclear Bragg peaks are marked as indexed in the trigonal HP-II phase. Broad temperature-dependent features indicative of short-range magnetic order are labeled in green. In dark orange are marked the positions of the magnetic order peaks which are described by $\mathbf{k}=(0,1,0)$ and $\mathbf{k}=\left(0,1, \frac{1}{2}\right)$ in the monoclinic symmetry of the HP-I phase for ease of comparison to earlier figures. These same magnetic structures, of course, have different indexing in the trigonal symmetry, but the $d$ spacings remain as shown. The inset shows the result of subtracting the $300 \mathrm{~K}$ data from the $80 \mathrm{~K}$ plotted against $Q-$ the SRO feature grows as temperature is decreased. The blue solid line through these data shows the RMC fit to this feature with spins constrained to be collinear (the fit used for the analysis) described in the text. Red and orange dashed lines show fits with unconstrained moment orientations, over two different $Q$ ranges, to show the robustness of the fit.

$Q$ typical of some kind of short-range magnetic order. This feature is reduced in magnitude upon increasing temperature but is not suppressed completely upon reaching room temperature. The $Q$ and temperature dependence of this feature, along with the lack of any similar observations in $\mathrm{x}$-ray diffraction studies, strongly suggest that it must be magnetic in origin - and, hence, local magnetic moments must be nonzero. Extrapolating the data taken while slowly warming suggests extinction of this feature at $360 \mathrm{~K}$, though, due to the long count times required to gain reliable statistics at this point, there is a high degree of uncertainty on this prediction. Certainly, whatever magnetic ordering this feature represents persists above room temperature. Additional peaks or features are also potentially seen at around $34.5^{\circ}$ and $39^{\circ}$ but without sufficient quality of statistics in the data to be certain as to a temperature dependence. These cannot be indexed with any reasonable magnetic vector in the HP-II unit cell-but neither can the large hump feature at low $Q$.

In order to characterize the broad short-range order (SRO) feature seen at the highest pressures, we carry out reverse Monte Carlo (RMC) refinements of spin configurations and fit the results to the data. In RMC refinement, a box (configuration) of spin vectors is generated. The spin orientations are initially random and are iteratively rotated-after each iteration, the expected scattering is calculated and compared with the experimental data, with the new configuration being accepted or rejected based on the quality of the comparison. Once the quality factor is hence optimized, the spin-spin correlation functions are calculated as an average over all spin pairs in the configuration. Repeating the refinement multiple times from differing initial seeds generates independent spin configurations and provides access to statistical uncertainties on spin correlation functions. Since the refinement is a stochastic process initialized from random spin arrangements, the final spin arrangements are the most disordered, consistent with the experimental data and other constraints imposed, namely, the assumed crystal structure and the requirement that every spin has equal magnitude $[52,53]$.

The calculations are carried out in the Spinvert program $[52,53]$. The data used are the result of subtracting the room temperature data at $183 \mathrm{kbar}$ from the $80 \mathrm{~K}$ data. The fits are restricted to the $Q$ region $0.56-1.50 \AA^{-1}$ to capture only the SRO feature and avoid the Bragg peaks and the artifacts of the pressure cell background. We verify the robustness of the fits by repeating them with multiple $Q$ ranges, and using both raw and temperature-subtracted data, and find no significant qualitative changes to the results. The temperature-subtracted data and several fits are shown in the inset in Fig. 6-good agreement is found over this range of $Q$, and the fits are robust to different conditions and ranges. 
The dashed orange and red lines in this inset show fits where the moment orientations are unconstrained. A slightly better fit is, however, found when the moments are constrained to lie along the $c$ axis, mimicking the Ising nature of the ambient-pressure magnetic order: suggesting that the collinear spin constraint improves the convergence of the refinements. This fit is shown as the solid blue line in the Fig. 6 inset and is the one used for subsequent analysis and discussion. This choice of fit is not seen to qualitatively impact the resulting correlation values-the fits appear to be rather robust to the chosen conditions. From this RMC fit, the spin correlation function could be extracted, $\langle\underline{S}(0) \cdot \underline{S}(r)\rangle$, which quantifies the extent of ordering between a magnetic site and each of its neighbors. These values are normalized such that perfect ferromagnetic correlations would give a value of 1 and perfect antiferromagnetic -1 . A value of 0 implies no net correlation between the two sites, i.e., a complete lack of order over that length scale. Figure 7 shows the extracted $\langle\underline{S}(0) \cdot \underline{S}(r)\rangle$ to a distance of $14 \AA$. The correlations decay quickly toward zero over this length scale, reflecting their short range. Fitting the values for $|\langle\underline{S}(0) \cdot \underline{S}(r)\rangle|$ with an exponentially decaying function gives characteristic correlation lengths of the order of $3 \AA$ for both inter- and intraplanar directions, which is similar to the distance between nearest neighbors. These two lengths being comparable is consistent with the system having become more three-dimensional in the HP-II phase-in a 2D system, one would expect much shorter correlation lengths along the interplanar direction. As the figure shows, the spin correlations found are predominantly antiferromagnetic, except for ferromagnetic nearest-neighbor interactions. This result appears remarkably similar to the ambientpressure $\mathbf{k}=\left(0,1, \frac{1}{2}\right)$ order-interplanar correlations are notably antiferromagnetic - but a short-ranged and disordered version of that ferromagnetic-chain arrangement. The simulated correlation values for such ideal long-range zigzag order with antiferromagnetic interplanar correlations are shown in the lower plot in Fig. 7, overlaid on the same data as the upper panel for comparison. Note that this magnetic order would be indexed as $\mathbf{k}=\left(0,1, \frac{1}{2}\right)$ in the monoclinic structural phases but is indexed as $\mathbf{k}=\left(0, \frac{1}{2}, \frac{1}{2}\right)$ in the trigonal HP-II structure found at this pressure. This result means that the interplanar order and correlations have gone from antiferromagnetic in the ambient state, to ferromagnetic in the HP-I phase, and then back to antiferromagnetic in the HP-II crystal structure. As exchange is weak between the crystal planes, even small changes in the crystalline environment can flip the sign of the interplanar exchange integrals in this system.

Normalizing the data using the nuclear Bragg profile for this fitting process additionally yields a resulting effective magnetic moment of $7.0 \mu_{B}$ per Fe ion, close to the ambient value of $5.7 \mu_{B}$ [54] within the sizable errors unavoidable in using this technique on high-pressure data. The
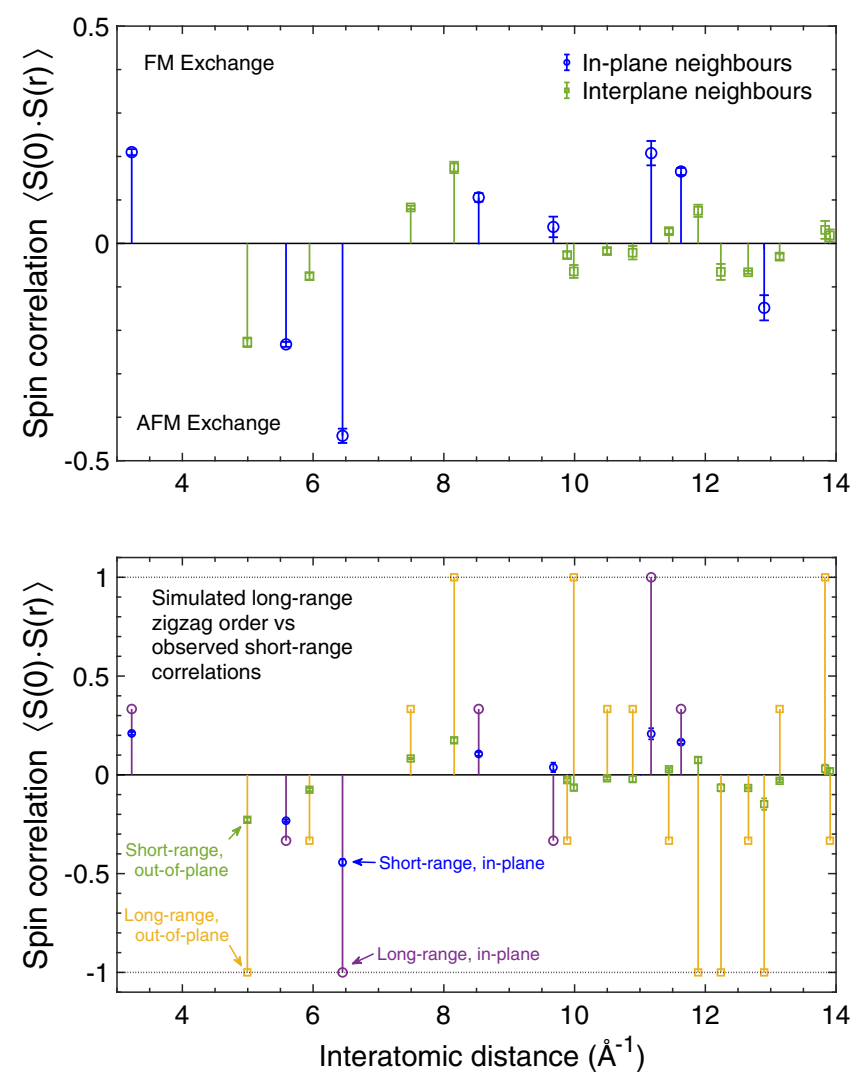

FIG. 7. Normalized spin-spin correlation function $\langle\underline{S}(0) \cdot \underline{S}(r)\rangle$ values for increasing interatomic distances from nearest-neighbor upward for $\mathrm{FePS}_{3}$ at $183 \mathrm{kbar}$ (upper plot). Perfect ferromagnetic correlations would give a value of +1 and perfect antiferromagnetic -1 . The values shown are extracted from the reverse Monte Carlo fits to the difference plot shown in the inset in Fig. 6. Nearest-neighbor correlations are seen to be ferromagnetic, and others predominantly antiferromagnetic, similar to those in ambient-pressure $\mathrm{FePS}_{3}$. The simulated correlation values for such ideal long-range zigzag order with antiferromagnetic interplanar correlations are shown overlaid on the same data in the lower plot for comparison.

systematic uncertainties are difficult to quantity exactly, as they are contributed to by imperfect background subtraction and absorption effects; the total moment in the SRO phase is, therefore, considered as an approximate estimate. Additionally, as the SRO is still present at $300 \mathrm{~K}$, the temperature subtraction underestimates the magnitude of the feature - the true extracted moment we estimate to be even larger. We also note that, although the RMC refinements are consistent with the existence of a unique collinear spin axis at high pressure, there is not a significant enough difference in the fit qualities to rule out the moments becoming isotropically oriented instead. This point warrants future investigation, as, if the moment orientations are found to not be constrained, the strong magnetic anisotropy that is present in $\mathrm{FePS}_{3}$ under ambient conditions is then absent at high pressures. 
The remarkable observation of persisting magnetism well into the metallic HP-II phase, with the Fe moment having a similar magnitude to that at ambient pressure, is in disagreement with the $\mathrm{x}$-ray emission spectroscopy results of Wang et al. [40], who report a spin crossover to an $S=0$ state at metallization. The temperature and wave vector dependence of the broad peak we observe in the highpressure neutron data, its absence in x-ray powder diffraction data, and the fit results provide strong evidence against any origin other than magnetism for this feature-we do not observe a collapse of the Fe spin configuration, in apparent contradiction with Wang et al.'s findings, as well as some theoretical predictions, such as those of Zheng et al. [55]. However, as outlined in Refs. [56,57], the x-ray spectra of the $K \beta \mathrm{Fe}^{\mathrm{II}}$ peak are dependent not only on the ionic spin state, but also heavily on the local environment, geometry, and surrounding bonds-including changes from insulating to metallic band structures. Further limits to the extraction of local moment from peak splitting as in this case are discussed in Ref. [58] — specifically, it is valid only when the charge-transfer satellite is negligible. As the HP-I to HP-II transition entails not only an insulator-metal transition but also a significant shift in the crystal structure, local environment, and dimensionality for the Fe ions, the change in the x-ray spectra seen by Wang et al. can potentially be explained by these effects rather than a loss of spin and atomic magnetic momentconsistent with our observation of magnetism in the high-pressure phase.

We additionally note that an unchanged local moment on the Fe site implies no valence or electron sharing changes on the Fe ions and precludes any mixtures of high and low spin domains or fractional occupancy. It then seems likely that another ionic site is responsible for the metallic conduction-almost certainly the $\mathrm{P}$ sites. Phosphorusphosphorus distances between the planes become significantly, abruptly, shorter in the HP-I to HP-II transition [35] to the point of reaching the typical length of a $\mathrm{P}-\mathrm{P}$ bond. Formations of such bonds and, hence, valence changes and carrier donation from the $\mathrm{P}$ rather than the Fe sites would seem a plausible explanation for a transition from insulating to metallic behavior while maintaining Fe local moments. This explanation is in agreement with the findings in the recent theoretical treatment of the insulator-metal transition by Evarestov and Kuzmin [59], which conclude that indeed phosphorus atoms are the main contributors to the conduction.

In summary, Fig. 8 displays the evolution of the principal magnetic features with pressure. Figure 8(a) plots against $d$ spacing the diffraction patterns in the vicinity of the magnetic peaks, normalized and with their vertical axis offset set to their corresponding pressure values. Two changes are clear-at first, the broad, asymmetric 2D-like peak spanning 010 and $01 \frac{1}{2}$ sharpens into a single, (a)
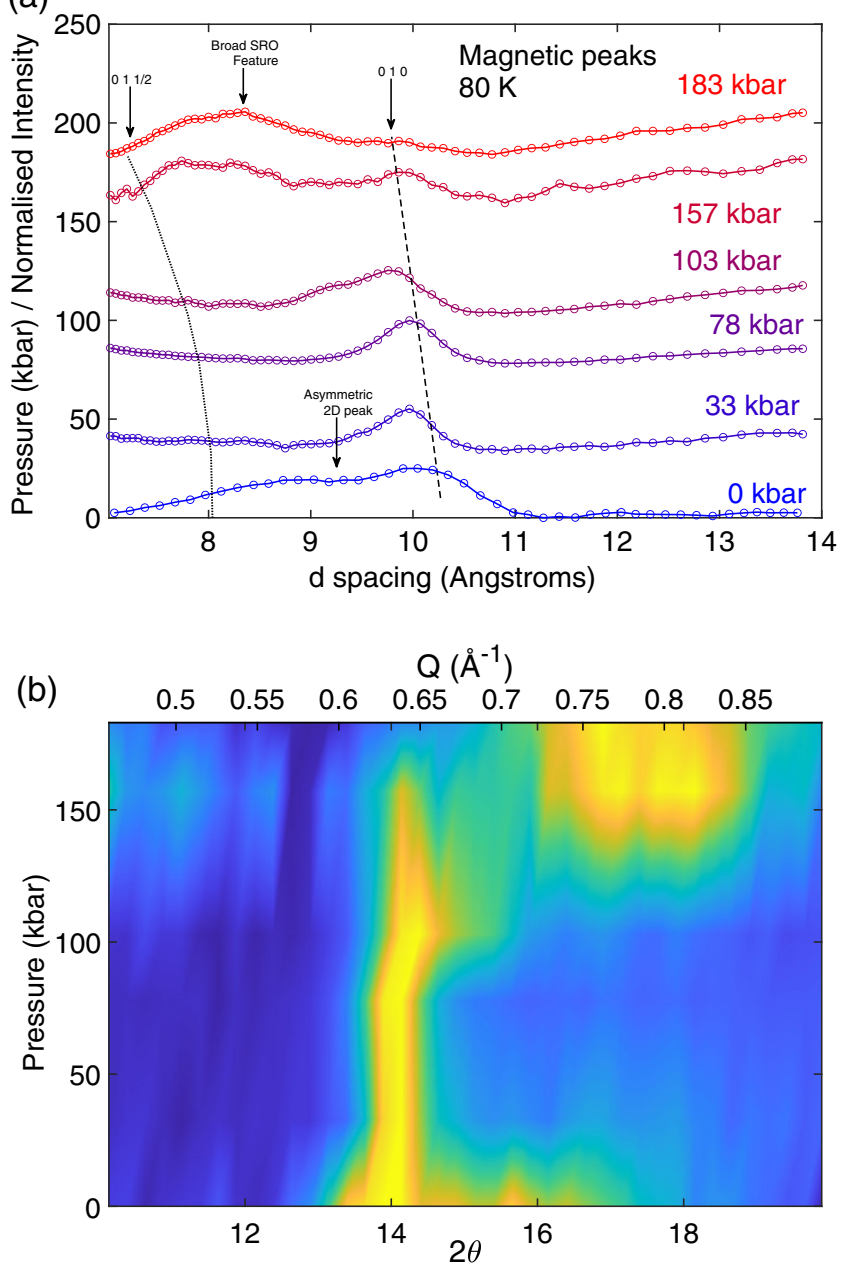

FIG. 8. (a) Magnetic peaks and features at $80 \mathrm{~K}$ for pressure points taken in sintered diamond cell on D20, ILL. The data are normalized and their vertical intercept set to their corresponding pressure values. The pressure-dependent $d$ spacings of the 010 and $01 \frac{1}{2}$ magnetic peak positions, extrapolated from the lattice parameters previously found for each pressure and indexed in the monoclinic space group, are shown as dashed and dotted lines, respectively. (b) Heat map of magnetic peaks at $80 \mathrm{~K}$ for sintered diamond pressures - color represents normalized intensity. At ambient pressure, the magnetic order is characterized by an asymmetric feature of both 010 and $01 \frac{1}{2}$ contributions. This feature then sharpens into a sharp 010 peak only by $20 \mathrm{kbar}$. The HP-I to HP-II structural transition can be seen around 150 kbar, which alters the magnetic state into a broad feature at higher $Q$ which persists above room temperature.

sharper, 3D-like Bragg peak at the 010 position. At higher pressures, this peak is suppressed, and a broad feature emerges at a lower $d$ spacing. The heat map shown in Fig. 8(b) clearly shows these two changes in behavior. The broad short-range-order feature at high $Q$ which emerges above around $130 \mathrm{kbar}$ is seen to coexist for a time with the 010 peak, one growing as the other is suppressed. This feature could, however, be due to pressure gradients and 
inhomogeneities within the sample region-a fraction of the sample may remain at an effective pressure below a transition the bulk has passed through. As the HP-I to HP-II structural transition is of strongly first-order character, if this change in magnetic order accompanies this transition, one would expect it to be correspondingly abrupt.

\section{DISCUSSION}

We measure the evolution of magnetic structure in Mottinsulating 2D honeycomb antiferromagnet $\mathrm{FePS}_{3}$ up to 183 kbar using neutron powder diffraction and construct the full pressure-temperature magnetic phase diagram, which corresponds well with previous structural studies. These results form the first example of dimensional tuning of magnetism in the crucial $T M \mathrm{PS}_{3}$ systems; they are likely to be common or interesting to compare for the rest of the family and to lead to better understanding of the novel insulator-metal transitions and states in these compounds. Knowledge of the precise structure and the magnetic order is additionally essential for informing theoretical and computational studies of such materials. Beyond even this rich family of compounds, this work forms a fundamental study of the evolution of low-dimensional magnetism and the formation of exotic short-range-ordered spin states in a near-ideal model system.

Two changes in behavior are observed. Initially, the ambient-pressure magnetic order (antiferromagnetic interplanar coupling with strong $2 \mathrm{D}$ character) is switched to a ferromagnetic interplanar ordering, with fully 3D-like sharp Bragg peaks. The in-plane structure of ferromagnetic chains, antiferromagnetically coupled, is seemingly preserved-leading to overall antiferromagnetic order. The Néel temperature is found through high-pressure dc magnetometry as well as neutron diffraction to increase roughly linearly with pressure, by $36 \mathrm{~K}$ over $100 \mathrm{kbar}$, with a change in slope at the first structural transition. These observations are consistent with the increased interplanar exchange expected as the crystal planes are forced closer together and the structure pushed into the HP-I phase.

The second change to the magnetism is a gradual suppression of this $\mathbf{k}=(0,1,0)$ order accompanied by an emergence of magnetic short-range order, with a transition or cutoff temperature seemingly above $300 \mathrm{~K}$. This change is indicated by a broad, roughly symmetric feature appearing at low $Q$ with strong temperature dependence. Reverse Monte Carlo fitting suggests this phase to be a short-ranged version of the original ambient-pressure structure-with a return to antiferromagnetic interplanar correlations. This short-range order in the HP-II structure indicates frustration of the magnetism. This frustration results from competition in magnetic exchange and from the details of anisotropy on magnetic sites.

The crossover to this short-range order state coincides with the previously observed HP-I to HP-II first-order structural transition and the corresponding insulator-metal transition in this material. The persistence of magnetism well into the HP-II metallic state is an observation in apparent contradiction with previous $\mathrm{x}$-ray spectroscopy results which suggest a spin crossover. We explain this discrepancy by suggesting the change in $\mathrm{x}$-ray emission spectra to be due to the dramatic changes to the local environment of the Fe ion through the HP-I to HP-II transition and metallization rather than a loss of spin state. Additionally, a transition to metallic behavior implies a change in character of any magnetism to a more itinerant nature-it is not trivial to connect the observed change to SRO to this expectation. More microscopic details of this SRO state and its impact on properties such as electrical transport await future investigation - it is, in fact, possible that the high-pressure superconducting state in sister material $\mathrm{FePSe}_{3}$ [40] borders a similar magnetic phase.

$\mathrm{FePSe}_{3}$ is directly comparable to $\mathrm{FePS}_{3}$ but with a lower electronic band gap and lower structural transition and metallization pressures-one would naively imagine due to the larger spatial influence of the Se sites allowing easier interplanar hopping. $\mathrm{FePSe}_{3}$ undergoes an equivalent insulator-metal transition to $\mathrm{FePS}_{3}$ at $70 \mathrm{kbar}$ and then enters a superconducting phase from $90 \mathrm{kbar}$ as pressure is further increased. It is reasonable to expect an equivalent state in $\mathrm{FePS}_{3}$ but also to expect this state to occur at higher pressures, or lower temperatures, than those yet measured. Conversely, the results we report on $\mathrm{FePS}_{3}$ are most likely to reflect on the understanding of $\mathrm{FePSe}_{3}$ - particularly, the spin crossover reported in both compounds at metallization we show not to be a valid interpretation of the results in the case of $\mathrm{FePS}_{3}$. Investigation of the magnetic and electronic properties of these materials as any superconductivity is approached is vital to understanding the characteristics of this state.

\section{CONCLUSIONS}

We have succeeded in mapping out the pressuretemperature magnetic phase diagram of van der Waals honeycomb antiferromagnet $\mathrm{FePS}_{3}$ : the first such study in the highly scrutinized $T M P X_{3}$ materials. We find an initial flip and continuous strengthening in the interplanar exchange, which we discuss in terms of the previously observed shear structural transition in these materials at low pressures and a tuning from 2D to 3D character. The susceptibility of the material to this change in the magnetism-mechanical grinding of the powder is seemingly enough to instigate it to some degree-is crucial information for designers of few-layer devices and heterostructures seeking to incorporate the magnetic properties of $T M \mathrm{P} X_{3}$, where strains and shear stresses are unavoidable. At higher pressures, where the material undergoes a collapse of the van der Waals gap and metallization, we observe a loss of long-range magnetic order and the emergence of a short-range-ordered magnetic state in its place, which survives to much higher temperatures. This 
result is counter to the previously suggested collapse of the magnetic moment and hugely important information to aid in understanding both the nature of the exotic metallic state found in this region of the phase diagram and potentially the formation of unconventional superconductivity at higher pressures. Classes of materials which demonstrate unconventional and high-temperature superconductivity such as the cuprates and pnictides have long attracted a great deal of interest and remain to be fully understood. Superconductivity as it exists in the simpler $T M \mathrm{PS}_{3}$ and $T M \mathrm{PSe}_{3}$ systems provides a valuable alternative mechanism to be explored, compared, and contrasted to these, to continue to develop our understanding of such emergent phenomena as a whole. An understanding of how magnetic order and the presence or absence of any valence transitions reinforces or competes with the formation of superconductivity in these compounds is a crucial piece of this puzzle.

Data presented in this paper resulting from the United Kingdom effort will be made available [60].

\section{ACKNOWLEDGMENTS}

The authors thank Suhan Son, Inho Hwang, Mark Dean, Gilbert Lonzarich, Emilio Artacho, Siân Dutton, Patricia Alireza, Shiyu Deng, Matt Tucker, and Mary-Ellen Donnelly for their generous help and discussions. This work was supported by the Institute for Basic Science (IBS) in Korea (Grant No. IBS-R009-G1). We also acknowledge support from Jesus College of the University of Cambridge and from the Engineering and Physical Sciences Research Council. The work was carried out with financial support from the Ministry of Education and Science of the Russian Federation in the framework of Increase Competitiveness Program of NUST MISiS (K2-2017-024). J.-G. P. was partially supported by the Leading Researcher Program of the National Research Foundation of Korea (Grant No. 2020R1A3B2079375). J. A. M. P.'s work at Oak Ridge National Laboratory (ORNL) was supported by the U.S. Department of Energy, Office of Science, Basic Energy Sciences, Materials Sciences and Engineering Division (analysis of magnetic diffuse scattering data) and by the Laboratory Directed Research and Development program of ORNL, managed by UT-Battelle, LLC for the U.S. Department of Energy (discussion of manuscript). This manuscript has been coauthored by UT-Battelle, LLC under Contract No. DE-AC05-00OR22725 with the U.S. Department of Energy. The United States Government retains and the publisher, by accepting the article for publication, acknowledges that the United States Government retains a nonexclusive, paid-up, irrevocable, worldwide license to publish or reproduce the published form of this manuscript, or allow others to do so, for United States Government purposes. The Department of Energy will provide public access to these results of federally sponsored research in accordance with the DOE Public Access Plan. J. A. M. P.'s preliminary work at Cambridge was supported by Churchill College, University of Cambridge. This research was supported by United Kingdom Research and Innovation Global Challenges Research Fund COMPASS Grant No. ES/P010849/1 and Cambridge Central Asia Forum, Jesus College, Cambridge. This project has received funding from the European Research Council (ERC) under the European Union's Horizon 2020 research and innovation program (Grant Agreement No. 681260).

[1] J.-G. Park, Opportunities and Challenges of 2D Magnetic van der Waals Materials: Magnetic Graphene?, J. Phys. Condens. Matter 28, 301001 (2016).

[2] P. Ajayan and K. Banerjee, Two-Dimensional van der Waals Materials, Phys. Today 69, No. 9, 38 (2016).

[3] N. Samarth, Condensed-Matter Physics: Magnetism in Flatland, Nature (London) 546, 216 (2017).

[4] Y. Zhou, H. Lu, X. Zu, and F. Gao, Evidencing the Existence of Exciting Half-Metallicity in Two-Dimensional $\mathrm{TiCl}_{3}$ and $\mathrm{VCl}_{3}$ Sheets, Sci. Rep. 6, 19407 (2016).

[5] K. S. Burch, D. Mandrus, and J.-G. Park, Magnetism in Two-Dimensional van der Waals Materials, Nature (London) 563, 47 (2018).

[6] V. Grasso and L. Silipigni, Low-Dimensional Materials: The $\mathrm{MPX}_{3}$ Family, Physical Features and Potential Future Applications, Riv. Nuovo Cimento 25, 6 (2002).

[7] R. Brec, Review on Structural and Chemical Properties of Transition Metal Phosphorous Trisulfides $\mathrm{MPS}_{3}$, Solid State Ionics 22, 3 (1986).

[8] B. L. Chittari, Y. Park, D. Lee, M. Han, A. H. MacDonald, E. Hwang, and J. Jung, Electronic and Magnetic Properties of Single-Layer $\mathrm{MPX}_{3}$ Metal Phosphorous Trichalcogenides, Phys. Rev. B 94, 184428 (2016).

[9] P. Rabu and M. Drillon, Layered Organic \& Inorganic Materials: A Way towards Controllable Magnetism, Adv. Eng. Mater. 5, 189 (2003).

[10] F. Wang, T. Shifa, P. Yu, P. He, Y. Liu, F. Wang, Z. Wang, X. Zhan, X. Lou, F. Xia, and J. He, New Frontiers on van der Waals Layered Metal Phosphorous Trichalcogenides, Adv. Funct. Mater. 28, 1802151 (2018).

[11] D. Lançon, H. C. Walker, E. Ressouche, B. Ouladdiaf, K. C. Rule, G. J. McIntyre, T. J. Hicks, H. M. Rønnow, and A. R. Wildes, Magnetic Structure and Magnon Dynamics of the Quasi-Two-Dimensional Antiferromagnet $\mathrm{FePS}_{3}$, Phys. Rev. B 94, 214407 (2016).

[12] G. Le Flem, R. Brec, G. Ouvard, A. Louisy, and P. Segransan, Magnetic Interactions in the Layer Compounds $\operatorname{MPX}_{3}(\mathrm{M}=\mathrm{Mn}, \mathrm{Fe}, \mathrm{Ni} ; \mathrm{X}=\mathrm{S}, \mathrm{Se})$, J. Phys. Chem. Solids 43, 455 (1982).

[13] K. Kurosawa, S. Saito, and Y. Yamaguchi, Neutron Diffraction Study on $\mathrm{MnPS}_{3}$ and $\mathrm{FePS}_{3}$, J. Phys. Soc. Jpn. 52, 3919 (1983).

[14] A. R. Wildes, S. J. Kennedy, and T. J. Hicks, True TwoDimensional Magnetic Ordering in $\mathrm{MnPS}_{3}$, J. Phys. Condens. Matter 6, L335 (1994). 
[15] A. R. Wildes, M. Harris, and K. W. Godfrey, TwoDimensional Critical Fluctuations in $\mathrm{MnPS}_{3}$, J. Magn. Magn. Mater. 177-181, 143 (1998).

[16] A. R. Wildes, B. Roessli, B. Lebech, and K. W. Godfrey, Spin Waves and the Critical Behaviour of the Magnetization in $\mathrm{MnPS}_{3}$, J. Phys. Condens. Matter 10, 6417 (1998).

[17] H. Rønnow, A. R. Wildes, and S. Bramwell, Magnetic Correlations in the $2 \mathrm{D}$ Honeycomb Antiferromagnet $\mathrm{MnPS}_{3}$, Physica (Amsterdam) 276B-278B, 676 (2000).

[18] K. Rule, S. Kennedy, D. Goossens, A. Mulders, and T. J. Hicks, Contrasting Antiferromagnetic Order between $\mathrm{FePS}_{3}$ and $\mathrm{MnPS}_{3}$, Appl. Phys. A 74, s811 (2002).

[19] K. Rule, T. Ersez, S. Kennedy, and T. J. Hicks, Identification of Features in the Powder Pattern of the Antiferromagnet $\mathrm{FePS}_{3}$ Using Polarization Analysis with Energy Analysis, Physica (Amsterdam) 335B, 6 (2003).

[20] A. R. Wildes, H. M. Rønnow, B. Roessli, M. J. Harris, and K. W. Godfrey, Static and Dynamic Critical Properties of the Quasi-Two-Dimensional Antiferromagnet $\mathrm{MnPS}_{3}$, Phys. Rev. B 74, 094422 (2006).

[21] K. C. Rule, G. J. McIntyre, S. J. Kennedy, and T. J. Hicks, Single-Crystal and Powder Neutron Diffraction Experiments on $\mathrm{FePS}_{3}$ : Search for the Magnetic Structure, Phys. Rev. B 76, 134402 (2007).

[22] A. R. Wildes, H. M. Rønnow, B. Roessli, M. J. Harris, and K. W. Godfrey, Anisotropy and the Critical Behaviour of the Quasi-2D Antiferromagnet, $\mathrm{MnPS}_{3}$, J. Magn. Magn. Mater. 310, 1221 (2007).

[23] K. C. Rule, A. R. Wildes, R. Bewley, D. Visser, and T. J. Hicks, High Energy Excitations Measured by Neutron Spectroscopy in $\mathrm{FePS}_{3}$, J. Phys. Condens. Matter 21, 124214 (2009).

[24] A. R. Wildes, K. C. Rule, R. I. Bewley, M. Enderle, and T. J. Hicks, The Magnon Dynamics and Spin Exchange Parameters of $\mathrm{FePS}_{3}$, J. Phys. Condens. Matter 24, 416004 (2012).

[25] A. R. Wildes, V. Simonet, E. Ressouche, G. J. McIntyre, M. Avdeev, E. Suard, S. A. J. Kimber, D. Lançon, G. Pepe, B. Moubaraki, and T. J. Hicks, Magnetic Structure of the Quasi-Two-Dimensional Antiferromagnet $\mathrm{NiPS}_{3}$, Phys. Rev. B 92, 224408 (2015).

[26] A. R. Wildes, V. Simonet, E. Ressouche, R. Ballou, and G. J. McIntyre, The Magnetic Properties and Structure of the Quasi-Two-Dimensional Antiferromagnet $\mathrm{CoPS}_{3}$, J. Phys. Condens. Matter 29, 455801 (2017).

[27] G. Ouvrard, R. Brec, and J. Rouxel, Structural Determination of Some $\mathrm{MPS}_{3}$ Layered Phases $(\mathrm{M}=\mathrm{Mn}$, Fe, Co, Ni and Cd), Mater. Res. Bull. 20, 1181 (1985).

[28] M. Friedel, Bull. Soc. Chim. Fr. 11, 115 (1894).

[29] M. Friedel, C.R. Hebd. Seances Acad. Sci. 119, 269 (1894).

[30] W. Klingen, G. Eulenberger, and H. Hahn, Über Hexathiound Hexaselenohypodiphosphate vom Typ $\mathrm{M}_{2}^{\mathrm{II}} \mathrm{P}_{2} \mathrm{X}_{6}$, Naturwissenschaften 55, 229 (1968).

[31] W. Klingen, Darstellung und Strukturbestimmung von Hexachalkogenohypodiphosphaten, Ph.D. thesis, Universität Hohenheim (LH), 1969.

[32] W. Klingen, G. Eulenberger, and H. Hahn, Über Hexachalkogeno-Hypodiphosphate vom Typ $\mathrm{M}_{2} \mathrm{P}_{2} \mathrm{X}_{6}$, Naturwissenschaften 57, 88 (1970).
[33] W. Klingen, R. Ott, and H. Hahn, Uber die Darstellung und Eigenschaften von Hexathio- und Hexaselenohypodiphosphaten, Z. Anorg. Allg. Chem. 396, 271 (1973).

[34] G. Ouvrard, R. Fréour, R. Brec, and J. Rouxel, A Mixed Valence Compound in the Two Dimensional $\mathrm{MPS}_{3}$ Family: $\mathrm{V}_{0.78} \mathrm{PS}_{3}$ Structure and Physical Properties, Mater. Res. Bull. 20, 1053 (1985).

[35] C. R. S. Haines, M. J. Coak, A. R. Wildes, G. I. Lampronti, C. Liu, P. Nahai-Williamson, H. Hamidov, D. Daisenberger, and S. S. Saxena, Pressure-Induced Electronic and Structural Phase Evolution in the van der Waals Compound $\mathrm{FePS}_{3}$, Phys. Rev. Lett. 121, 266801 (2018).

[36] M. J. Coak, S. Son, D. Daisenberger, H. Hamidov, C. R. S. Haines, P. L. Alireza, A. R. Wildes, C. Liu, S. S. Saxena, and J.-G. Park, Isostructural Mott Transition in 2D Honeycomb Antiferromagnet $\mathrm{V}_{0.9} \mathrm{PS}_{3}$, npj Quantum Mater. 4, 38 (2019).

[37] M. J. Coak, Y.-H. Kim, Y. S. Yi, S. Son, S. K. Lee, and J.-G. Park, Electronic and Vibrational Properties of the TwoDimensional Mott Insulator $\mathrm{V}_{0.9} \mathrm{PS}_{3}$ under Pressure, Phys. Rev. B 100, 035120 (2019).

[38] D. M. Jarvis, Ph.d. Thesis, University of Cambridge, 2020, https://doi.org/10.17863/CAM.60324.

[39] M. J. Coak, D. M. Jarvis, H. Hamidov, C. R. S. Haines, P. L. Alireza, C. Liu, S. Son, I. Hwang, G. I. Lampronti, D. Daisenberger, P. Nahai-Williamson, A. R. Wildes, S. S. Saxena, and J.-G. Park, Tuning Dimensionality in van-derWaals Antiferromagnetic Mott Insulators TMPS 3 , J. Phys. Condens. Matter 32, 124003 (2020).

[40] Y. Wang, J. Ying, Z. Zhou, J. Sun, T. Wen, Y. Zhou, N. Li, Q. Zhang, F. Han, Y. Xiao, P. Chow, W. Yang, V. V. Struzhkin, Y. Zhao, and H.-K Mao, Emergent Superconductivity in an Iron-Based Honeycomb Lattice Initiated by Pressure-Driven Spin-Crossover, Nat. Commun. 9, 1914 (2018).

[41] T. C. Hansen, P. F. Henry, H. E. Fischer, J. Torregrossa, and P. Convert, The D20 Instrument at the ILL: A Versatile High-Intensity Two-Axis Neutron Diffractometer, Meas. Sci. Technol. 19, 034001 (2008).

[42] S. Klotz, T. Sträsle, G. Rousse, G. Hamel, and V. Pomjakushin, Angle-Dispersive Neutron Diffraction under High Pressure to 10 GPa, Appl. Phys. Lett. 86, 031917 (2005).

[43] S. S. Saxena, M. J. Coak, S. E. Dutton, C. R. S. Haines, H. Hamidov, T. Hansen, D. M. Jarvis, C. Liu, and A. R. Wildes, ILL Experiment 5-31-2545 on D20 (2018), https://doi.org/ 10.5291/ill-data.5-31-2545.

[44] S. Klotz, T. Sträsle, B. Lebert, M. d'Astuto, and T. Hansen, High Pressure Neutron Diffraction to beyond $20 \mathrm{GPa}$ and below $1.8 \mathrm{~K}$ Using Paris-Edinburgh Load Frames, High Press. Res. 36, 73 (2016).

[45] M. J. Coak, S. E. Dutton, C. R. S. Haines, H. Hamidov, T. Hansen, D. M. Jarvis, S. Klotz, C. Liu, J.-G. Park, S. S. Saxena, and A. R. Wildes, ILL Experiment 5-31-2646 on D20 (2019), https://doi.org/10.5291/ill-data.5-31-2646.

[46] W. G. Marshall and D. J. Francis, Attainment of NearHydrostatic Compression Conditions Using the Paris Edinburgh Cell, J. Appl. Crystallogr. 35, 122 (2002).

[47] T. Strässle, S. Klotz, K. Kunc, V. Pomjakushin, and J. S. White, Equation of State of Lead from High-Pressure Neutron Diffraction up to 8.9 GPa and Its Implication 
for the NaCl Pressure Scale, Phys. Rev. B 90, 014101 (2014).

[48] V. Aksenov, A. Balagurov, V. Glazkov, D. Kozlenko, I. Naumov, B. Savenko, D. Sheptyakov, V. Somenkov, A. Bulkin, V. Kudryashev, and V. Trounov, DN-12 Time-ofFlight High-Pressure Neutron Spectrometer for Investigation of Microsamples, Physica (Amsterdam) 265B, 258 (1999).

[49] V. A. Somenkov, High-Pressure Neutron Scattering over the Ages, J. Phys. Condens. Matter 17, S2991 (2005).

[50] H. Mao, J. Xu, and P. Bell, Calibration of the Ruby Pressure Gauge to 800 kbar under Quasi-Hydrostatic Conditions, J. Geophys. Res. 91, 4673 (1986).

[51] J. Rodriguez-Carvajal, Recent Advances in Magnetic Structure Determination by Neutron Powder Diffraction, Physica (Amsterdam) 192B, 55 (1993).

[52] J. A. M. Paddison and A. L. Goodwin, Empirical Magnetic Structure Solution of Frustrated Spin Systems, Phys. Rev. Lett. 108, 017204 (2012).

[53] J. A. M. Paddison, J. R. Stewart, and A. L. Goodwin, Spinvert: A Program for Refinement of Paramagnetic Diffuse Scattering Data, J. Phys. Condens. Matter 25, 454220 (2013).
[54] P. A. Joy and S. Vasudevan, Magnetism in the Layered Transition-Metal Thiophosphates $\mathrm{MPS}_{3}(\mathrm{M}=\mathrm{Mn}, \mathrm{Fe}$, and Ni), Phys. Rev. B 46, 5425 (1992).

[55] Y. Zheng, X.-x. Jiang, X.-x. Xue, J. Dai, and Y. Feng, Ab Initio Study of Pressure-Driven Phase Transition in $\mathrm{FePS}_{3}$ and $\mathrm{FePSe}_{3}$, Phys. Rev. B 100, 174102 (2019).

[56] G. Vankó, T. Neisius, G. Molnár, F. Renz, S. Kárpáti, A. Shukla, and F. M.F. de Groot, Probing the 3d Spin Momentum with X-Ray Emission Spectroscopy: The Case of Molecular-Spin Transitions, J. Phys. Chem. B 110, 11647 (2006).

[57] J.-P. Rueff, C.-C. Kao, V. V. Struzhkin, J. Badro, J. Shu, R. J. Hemley, and H. K. Mao, Pressure-Induced High-Spin to Low-Spin Transition in FeS Evidenced by X-Ray Emission Spectroscopy, Phys. Rev. Lett. 82, 3284 (1999).

[58] S.-J. Oh, G.-H. Gweon, and J.-G. Park, Origin of $3 \mathrm{~s}$ Splittings in the Photoemission Spectra of $\mathrm{Mn}$ and $\mathrm{Fe}$ Insulating Compounds, Phys. Rev. Lett. 68, 2850 (1992).

[59] R. A. Evarestov and A. Kuzmin, Origin of PressureInduced Insulator-to-Metal Transition in the van der Waals Compound $\mathrm{FePS}_{3}$ from First-Principles Calculations, J. Comput. Chem. 41, 14 (2020).

[60] https://wrap.warwick.ac.uk/143817. 